\title{
on "Evaluation of the effectiveness of a
}

Oskar Rosiak* and Magdalena Jozefowicz-Korczynska

Department of Otolaryngology, Balance Disorders Unit, Medical University of Lodz, The Norbert Barlicki Memorial Teaching Hospital, Lodz, Poland

Dear Editor,

We would like to express our sincere gratitude to Miracelli et al. for their interest in our research and we would like to elaborate on the use of Head Mounted Displays (HMD) in vestibular rehabilitation.

A key concept in virtual reality is "immersion" a sense of physical "self" in a non-physical, artificial world. Achieving high immersion requires multisensory stimulation. Out of the different stimuli the most important seems to be visual input delivered in high detail, realistic graphics. Application of HMDs to virtual reality experience greatly improves immersion, because it creates a stronger sensory mismatch between the vestibular system and visual input. However, it has been reported that higher detail visual realism in HMDs causes more cybersickness symptoms like headaches, dizziness, nausea and eye stress [1]. In time these symptoms alleviate [2, 3], which is most likely caused by vestibular compensation mechanisms and a shift from visual dependence to proprioceptive and vestibular input predominance. It may be hypothesized that more visually dependent

${ }^{*}$ Corresponding author: Oskar Rosiak, Balance Disorders Unit, Medical University of Lodz, Ul. Kopcinskiego 22 90-153 Lodz, Poland. Tel.: +48 501063456; E-mail: orosiak@me.com. patients are prone to cybersickness, but this requires further investigation. The issue of cybersickness in wearable devices for virtual reality is a subject of study, and it has been reported that dynamic focus of the visual field is an important factor in symptom presentation [2]. Nonetheless, around $20 \%$ to $80 \%$ of the population experience cybersickness in VR environments [4].

In our study [5], VR was displayed on a flat screen 2D display. None of the patients complained of cybersickness because they could locate external reference points for their visual input. We are aware of the limitations of this approach, where application of HMDs would further enhance the sensory conflict [3] and possibly cause a greater improvement in symptoms, but also decrease patients' tolerance of the VR therapy due to possible cybersickness. Moreover, a VR system based only on HMD allows the clinician to track only head and eye movements, whereas a hybrid system as described in our study can record the motion of extremities and COP displacement.

We would like to propose an alternative, novel approach, which utilizes Augmented Reality (AR). In this interactive environment computer generated perceptual information overlays with the physical world "augmenting" the sensory input. In comparison 
to $\mathrm{VR}, \mathrm{AR}$ does not completely replace the visual information and maintains external reference points, which prevent strong motion sickness. Visual AR can be delivered by different display measures - in one of our exercises - The Meteorites - a patient's own posture was projected into the virtual world. This is a simplified approach to AR, which can be improved by the application of Head Mounted Displays to a hybrid unit as described in our study.

This new concept in rehabilitation requires further investigation and we hope that a multi-centered research study would be possible in the near future.

\section{References}

[1] T. Arttu, Effect of Visual Realism on Cybersickness in Virtual Reality, (2018) 1-49.

[2] T.M. Porcino, E. Clua, D. Trevisan, C.N. Vasconcelos and L. Valente, Minimizing cyber sickness in head mounted display systems: Design guidelines and applications, in: 2017 IEEE 5th Int Conf Serious Games Appl Heal SeGAH 2017, 2017: pp. 1-11. doi:10.1109/SeGAH.2017.7939283

[3] A. Micarelli, A. Viziano, I. Augimeri, D. Micarelli and M. Alessandrini, Three-dimensional head-mounted gaming task procedure maximizes effects of vestibular rehabilitation in unilateral vestibular hypofunction: A randomized controlled pilot trial, Int J Rehabil Res (2017). doi:10.1097/MRR.0000000000000244

[4] L. Rebenitsch and C. Owen, Review on cybersickness in applications and visual displays, Virtual Real (2016). doi:10.1007/s10055-016-0285-9

[5] O. Rosiak, K. Krajewski, M. Woszczak and M. JozefowiczKorczynska, Evaluation of the effectiveness of a Virtual Reality-based exercise program for Unilateral Peripheral Vestibular Deficit, J Vestib Res (2019). doi:10.3233/ves180647 